\title{
Providing Explicit Information Disrupts Implicit Motor Learning After Basal Ganglia Stroke
}

\author{
Lara A. Boyd ${ }^{1,3}$ and Carolee J. Winstein ${ }^{2}$ \\ ${ }^{1}$ Department of Physical Therapy and Rehabilitation Sciences, Department of Neurology, University of Kansas Medical Center, \\ Kansas City, Kansas 66160, USA; ${ }^{2}$ Department of Biokinesiology and Physical Therapy, Department of Neurology, University of \\ Southern California, Los Angeles, California 90033, USA
}

\begin{abstract}
Despite their purported neuroanatomic and functional isolation, empirical evidence suggests that sometimes conscious explicit processes can influence implicit motor skill learning. Our goal was to determine if the provision of explicit information affected implicit motor-sequence learning after damage to the basal ganglia. Individuals with stroke affecting the basal ganglia (BG) and healthy controls $(\mathrm{HC})$ practiced a continuous implicit motor-sequencing task; half were provided with explicit information (EI) and half were not (No-EI). The focus of brain damage for both BG groups was in the putamen. All of the El participants were at least explicitly aware of the repeating sequence. Across three days of practice, explicit information had a differential effect on the groups. Explicit information disrupted acquisition performance in participants with basal ganglia stroke but not healthy controls. By retention (day 4), a dissociation was apparent-explicit information hindered implicit learning in participants with basal ganglia lesions but aided healthy controls. It appears that after basal ganglia stroke explicit information is less helpful in the development of the motor plan than is discovering a motor solution using the implicit system alone. This may be due to the increased demand placed on working memory by explicit information. Thus, basal ganglia integrity may be a crucial factor in determining the efficacy of explicit information for implicit motor-sequence learning.
\end{abstract}

Explicit learning and memory are neuroanatomically sub-served by discrete regions within the medial temporal lobe that support conscious recollection of events and facts. Because no single lesion completely eliminates the development of procedural memories (although many severely disrupt them) the identification of the particular neuroanatomic structures that mediate implicit memory is not entirely clear. Studies of individuals with local damage (Vakil et al. 2000) or degenerative disease (Doyon et al. 1997) demonstrate that the basal ganglia are one of the regions that support implicit motor-sequence learning.

\section{Implicit Learning After Brain Damage}

Implicit learning of regularities within sequences of action has been demonstrated in healthy nonneurologically impaired individuals (Nissan and Bullemer 1987; Wulf and Schmidt 1997; Reber and Squire 1998; Scoville and Milner 2000) as well as in persons with damage in the medial temporal lobe in structures associated with explicit memory (Glisky and Schacter 1987; Nissan and Bullemer 1987; Reber and Squire 1998; Scoville and Milner 2000). However, less work has focused on implicit learning capability following unilateral damage to the neural regions supporting the implicit memory system (Gomez-Beldarrain et al. 1998; Vakil et al. 2000; Boyd and Winstein 2001, 2003; Pohl et al. 2001).

Most studies examining the role of the basal ganglia in implicit motor sequence learning have considered individuals with degenerative disease such as Parkinson's disease (PD; PascualLeone et al. 1993; Jackson et al. 1995; Knowlton et al. 1996; Doyon et al. 1997, 1998; Vakil et al. 2000). Because dopamine depletion severely reduces the outflow from the basal ganglia,

\section{${ }^{3}$ Corresponding author.}

E-MAIL Lboyd@kumc.edu; FAX (913) 588-4568.

Article and publication are at http://www.learnmem.org/cgi/doi/10.1101/ Im.80104. and in turn may hamper the function of other dopaminergic systems in the brain, it is possible that deficits recorded in people with PD might be partially the result of altered neural function in regions of the brain outside of the basal ganglia. Thus, this approach may not clearly identify the role of the basal ganglia during implicit motor learning (Agid 1993; Kastner et al. 1993; Holthoff-Detto et al. 1997).

Attempting to minimize the potential effects of widespread brain damage that may be associated with degenerative disease, other work has examined implicit motor learning after stroke. Two studies (Boyd and Winstein 2001; Pohl et al. 2001) demonstrated implicit motor-sequence learning following unilateral stroke but could not address regional contributions to learning, because they lacked specific information regarding the location and extent of brain damage. In the current research we addressed this shortcoming by examining only individuals with stroke affecting the basal ganglia. To date only one study has examined the impact of unilateral basal ganglia damage (Vakil et al. 2000), concluding that implicit motor sequence learning was impaired as compared to age-matched healthy controls. However, all participants used their right hand for serial reaction time task practice (a unimanual task), resulting in five of 16 participants using their stroke-affected arm. This is problematic, as use of the hemiparetic upper extremity for some but not all participants likely biased response times and may have inflated between-group differences.

Inherently difficult in a stroke model is the necessity of disentangling motor execution impairments associated with the affected hemibody from deficient motor learning. Requiring individuals with stroke to use the more involved, contralesional upper extremity for task practice is problematic; differences between stroke and control groups might be inflated by impaired motor execution, which in turn, could mask motor learning. Severely affected individuals, with poor motor ability, might be 
unable to perform the experimental task and be excluded from study. We minimized this problem by requiring participants with basal ganglia stroke to practice our implicit motor learning task using the less involved, ipsilesional upper extremity and matching healthy controls for arm use.

Previous work has established that the motor control of both upper extremities may be disrupted by unilateral stroke (Pohl et al. 1997; Winstein et al. 1999); however, the conclusions of motor learning studies have been less consistent. Some have found motor learning deficits (Boyd and Winstein 2003), while others have not (Gomez-Beldarrain et al. 1998; Winstein et al. 1999). Taken together, these findings led us to ask whether basal ganglia stroke would have an effect on motor learning of an implicit task (continuous-tracking) when participants used their ipsilesional upper extremity, and if so, how?

\section{Putative Roles of the Basal Ganglia During Implicit Learning}

Evidence suggests that the basal ganglia are important for advance preparation of responses in learned sequences of action (Jennings 1995; Harrington and Haaland 1998). When given knowledge about upcoming responses, healthy control participants use advance information to prepare not only their first, but also subsequent movements. In contrast, individuals with PD only use advance information to prepare their initial response. These data pose an important question: Will individuals with unilateral stroke affecting the basal ganglia take advantage of explicit information to improve implicit motor-sequence learning through advance planning?

Other data suggest that basal ganglia damage also impacts performance of tasks with working memory components. Data from individuals with PD show that degeneration of the basal ganglia disrupts working memory (Owen 1997). This finding might be explained by the secondary effects of dopamine depletion which may act on the prefrontal cortex via the reciprocal loops from the basal ganglia (Middleton and Strick 1997). Neuroimaging work clarifies this relationship demonstrating that caudate activity can be tied to working memory load (Braver et al. 1997), and suggesting the engagement of a fronto-striatal network whenever working memory is taxed (Klingberg et al. 1997; Poldrack et al. 1999). Taken together these data suggest the possibility that basal ganglia activity may be linked to the demands placed on working memory during motor learning, perhaps integrating cognitive information into ongoing action.

\section{Influence of Explicit Information on Implicit Learning}

The influence of explicit information of implicit learning has been disparately reported in the scientific literature (Reber 1976; Green and Flowers 1991; Curran and Keele 1993; Reber and Squire 1998; Boyd and Winstein 2001, 2003; Shea et al. 2001). Some investigators report beneficial effects of explicit information on implicit motor learning (Curran and Keele 1993; Boyd and Winstein 2001), others detrimental effects (Reber 1976; Green and Flowers 1991), and others little consequence (Reber and Squire 1998; Shea et al. 2001). These seemingly contradictory findings may result from combined factors such as task differences, the type, timing and salience of explicit information, and participant characteristics. Further, very few (Vakil et al. 2000; Boyd and Winstein 2003) have considered the impact of explicit information on implicit motor sequence learning after regional brain damage caused by stroke. Interestingly, evidence is accumulating that in some cases adoption of conscious strategies for movement disrupts motor learning (Reber 1976; Green and Flowers 1991; Verdolini-Marston and Balota 1994; Winstein et al. 1996; Boyd and Winstein 2003).
Thus, there were two purposes to this study. First, we sought to assess the effect that providing explicit information would have on learning an implicit motor skill. Participants underwent 3 days of continuous-tracking (CT) task practice where the middle third of each tracking trial was repeated; the first and last thirds of each trial were random. On day 4 a retention test was given to assess implicit learning. Explicit information (EI) of the repeating sequence was provided to half of the participants, while the other half practiced without explicit information (NoEI). Second, we examined the impact of unilateral basal ganglia damage on both implicit motor-sequence learning and the ability to exploit explicit information during learning. We expected that explicit information would benefit implicit motor-sequence learning in healthy, neurologically intact (HC) individuals and in participants with unilateral stroke affecting the basal ganglia (BG). Our data revealed that we were only partially correct; EI benefited the HC group but disrupted implicit motor learning in participants with basal ganglia damage.

\section{RESULTS}

\section{Lesion Location}

The focus of lesion overlap for both BG EI and BG No-EI groups was the putamen (Fig. 1; Talairach coordinates BG EI -21, 1, 12; BG No-EI -21, 6, 11; Talairach and Tournoux 1988).

\section{Tracking Error}

\section{Acquisition Performance}

All participants in this study were able to decrease tracking error on the repeated sequence across acquisition practice (Fig. 2A,B). However, examination of our data showed that contrary to our hypothesis, individuals in the BG group did not benefit from explicit information. A full-factor ANOVA (with repeated measures correction) confirmed this observation via a three-way Group (HC, BG) by Information (EI, No-EI) by Day $(1,2,3)$ interaction $\left(F_{(2,32)}=4.76, P=0.04\right)$. Post hoc analyses revealed that explicit information had the opposite effect on BG compared to the HC groups.

When the information factor was evaluated separately for each group, (Group by Day ANOVA), performance of the two EI groups (HC, BG) were significantly different $\left(F_{(2,16)}=4.82\right.$, $P=0.05)$, but the No-EI groups were not $(P=0.19)$. This was due to the poorer performance of the BG EI group compared to the HC EI group (Fig. 2A,B).

Separate consideration of the two groups (Information by Day ANOVA) showed the differential effect of explicit information on acquisition performance. Although both HC information groups improved their performance with practice (indexed by decreased tracking error; Main Effect of Block $F_{(2,16)}=6.55$, $P=0.008$; Fig. 2A) there was not an effect of information $(P=0.206)$. This suggests a limited effect of explicit information during acquisition practice for the HC group. In contrast, a significant Information by Day interaction was found for the BG groups' acquisition performance $\left(F_{(2,16)}=3.62, P=0.05\right.$; Fig. $\left.2 \mathrm{~B}\right)$. Post hoc evaluation demonstrated that the BG groups (EI, No-EI) were similar at the end of day $1(P=0.41)$, trended towards difference at the end of day $2(P=0.09)$, and were reliably different at the end of day $3\left(F_{(1,8)}=18.37, P=0.01\right)$. This finding suggests that providing EI disrupted implicit motor learning as indexed by tracking error in participants with BG stroke.

\section{Retention Test}

At retention we noted significant differences in implicit learning that were dependent on the presence of basal ganglia damage and explicit instruction (Group by Information interaction; 


\section{Explicit Information}

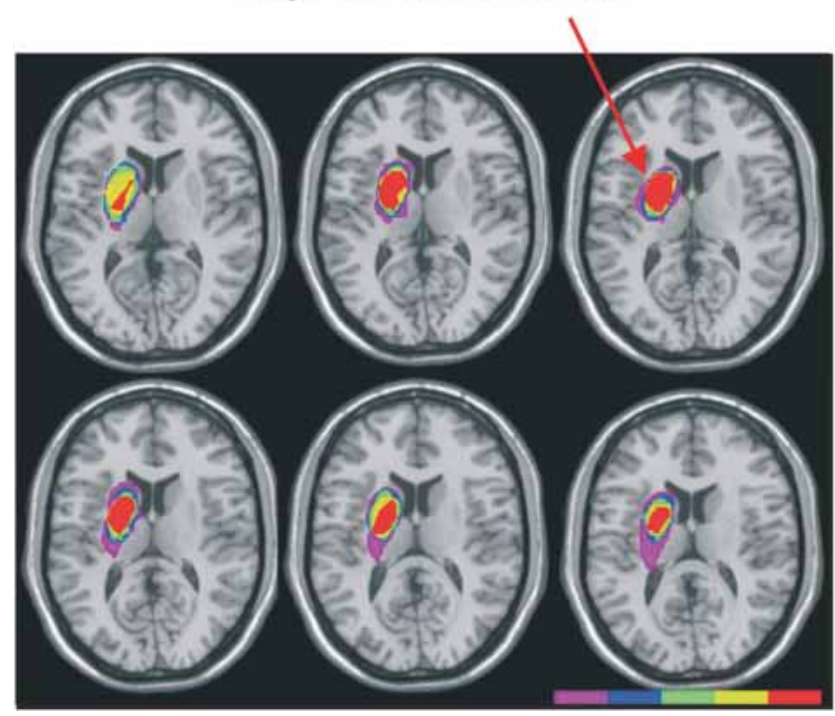

No Explicit Information

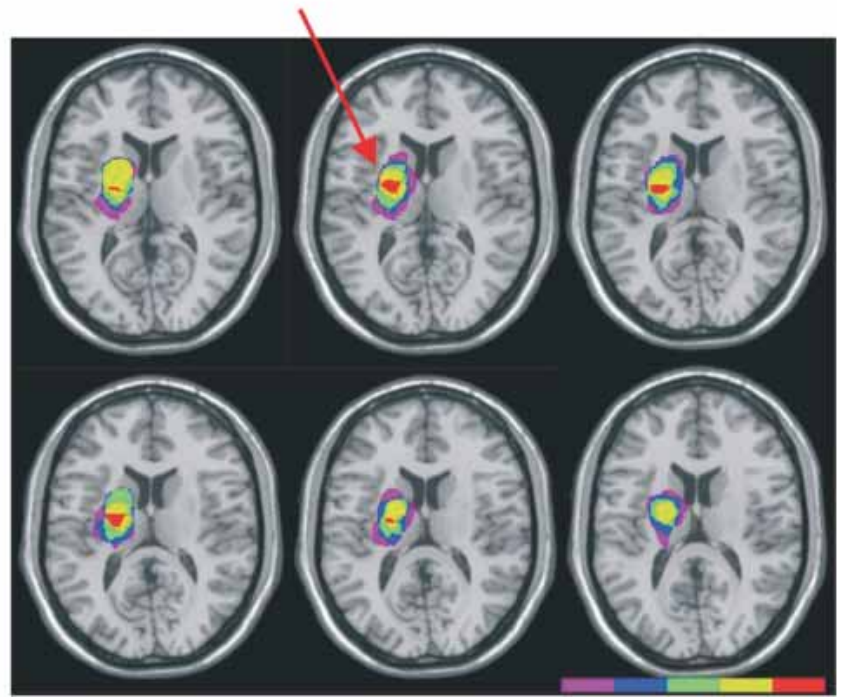

Figure 1 Representations of strokes reconstructed by transcribing lesions from MRI or CT scans onto axial templates using MRIcro software (Rorden 2003). All lesions were reconstructed on the left for illustration purposes. Standard slices are 2-mm thick and displayed from superior (top left) to inferior (bottom right). The color bar represents overlap density (violet = no overlap [0\%], red = maximal overlap [100\%]). The focus of overlap for the BG El group was in the putamen (Talairach coordinates - 21, 6, 11); the focus of overlap for the BG No-El group was also in the putamen (Talairach coordinates $-21,1,12$ ).

$F_{(1,16)}=6.50, P=0.03$; Fig. 3$)$. Post hoc tests demonstrated that this interaction was the result of differences between the HC EI and HC No-EI groups $\left(F_{(1,8)}=8.60, P=0.02\right)$. There was a trend for a similar difference between the BG EI and BG No-EI groups $(P=0.08)$. Inspection of retention test data revealed a greater change in tracking errors for the BG No-EI than the BG EI group. The opposite pattern was seen for the HC groups (HC EI showed greater tracking error change than the HC No-EI). This finding suggests that explicit information benefited implicit CT learning in individuals without neurologic damage but impaired implicit CT learning for participants with basal ganglia damage.

\section{Time Lag of Tracking}

\section{Acquisition Performance}

Reductions in the time lag of tracking were evident for all groups; however, the HC EI was significantly faster than the HC No-EI group suggesting that providing explicit information allowed for advance preparation and faster responses in neurologically intact participants (Fig. 4A). Across acquisition, both HC information groups were able to reduce time lag of tracking (Main Effect of Block $\left.F_{(2,16)}=3.61, P=0.05\right)$. However, this decrease was larger for the HC EI group than the HC No-EI group as evidenced by the Main Effect of Information $\left(F_{(1,8)}=13.74, P=0.01\right)$. Both BG groups demonstrated the ability to decrease their time lag on the CT task from 214 to 188 ms for the BG EI group and from 214 to $172 \mathrm{~ms}$ for the BG No-EI group (Main Effect of Block $\left.F_{(2,16)}=10.09, P=0.001\right)$. Time lag of tracking, however, was not significantly affected by the provision of explicit information (Main Effect of Information $P=0.714$; Fig. 4B).

\section{Retention Test}

A significant Group by Information interaction at the retention test $\left(F_{(1,16)}=4.97, P=0.04\right)$ demonstrated differences in learning to predict the pattern of the target (Fig. 4A,B, far right bars). Follow-up tests revealed that the locus of this interaction was the superior performance of the HC EI group compared to the HC No-EI group $\left(F_{(1,8)}=8.60, P=0.03\right)$. Observation of the data showed that the HC EI group had a much shorter time lag of tracking at retention (HC EI 109 ms; HC No-EI 172 ms). In contrast, providing explicit information had little effect on time lag of tracking for the BG EI compared to the BG No-EI group $(P=0.40 ;$ BG EI time lag $=160, \mathrm{BG}$ No-EI $=144 \mathrm{~ms})$.

\section{Tracking Accuracy}

\section{Acquisition Performance}

All groups demonstrated improvements in tracking accuracy across practice regardless of information condition. There was no statistically reliable effect of information or group on tracking accuracy across acquisition (Fig. 5A,B).

\section{Retention Test}

All groups also showed improved tracking accuracy at the retention test. No significant differences existed between group or information conditions at retention (Fig. 5A,B, far right bars).

\section{Explicit Information}

\section{No-EI Groups}

Explicit testing for the HC No-EI group performed after the retention test (day 4) demonstrated that none of the HC participants had subjective awareness of a repeating sequence. When prompted by the investigator, only one participant correctly located the repeating sequence in the middle third of the tracking pattern. Recognition memory was scored as percentage correct. A numeric score of $50 \%$ or less represents no recognition; whereas above 50\% demonstrates increasing recognition with higher scores. Recognition memory was slightly better than chance (above 50\%) in the HC No-EI group (Table 1A). Similarly, the BG No-EI group did not gain explicit information across the three days of practice. Recognition of the repeated sequence for the $B G$ No-EI group was below chance $(<50 \%)$.

\section{EI Groups}

At the end of day 1 of practice explicit testing revealed no subjective awareness of the repeating sequence for the HC EI group.

\section{Learning \& Memory}


A

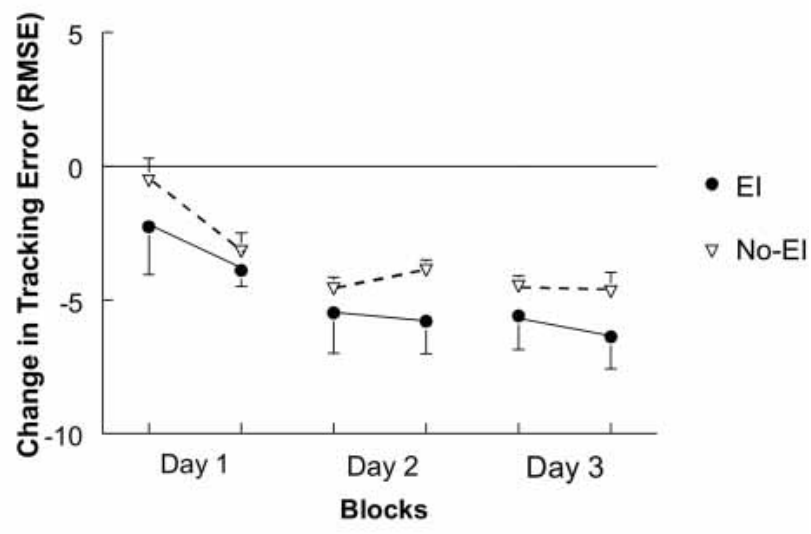

B

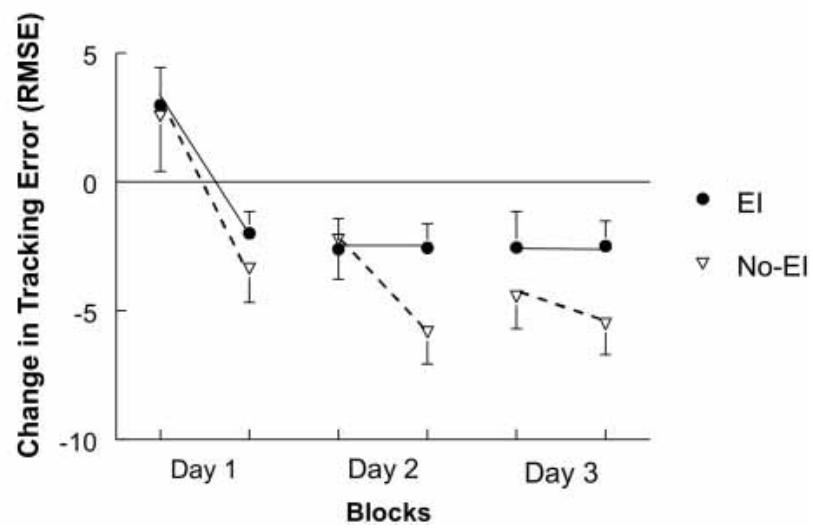

Figure 2 Change in tracking error for the groups across three days of practice. The zero line reflects performance on random tracking sequences. Data below this line reflects reductions (improvements) in tracking error for repeated sequences relative to random pattern tracking. Error bars are standard error of the mean (SEM). El group is closed lines and symbols, No-El group is dashed lines and open symbols. $(A)$ Healthy Control Groups. Both groups (HC El and No-El) benefited from practice regardless of the presence of explicit information. (B) Basal Ganglia Groups. Across practice both BG El and No-El showed decreased tracking error. However, the BG El group's performance was poorer (less change) than that of the No-El group's.

When prompted, only one participant guessed correctly that the middle third was the repeated element; recognition was below chance (Table 1B). After being explicitly provided with partial information about the sequence on days 2 and 3, all HC EI participants were subjectively aware of a repeating sequence. Despite this information, at the conclusion of day 2 , recognition was at chance $(46 \%)$. The pretest on day 3 revealed that recognition memory was $60 \%$ and practice improved this to $73 \%$ (Table $1 \mathrm{C}$ ).

Explicit knowledge was similarly poor for the BG EI group at the conclusion of day 1 . None of the BG EI participants noticed the sequence, and only one guessed the correct location of the repeated elements when prompted. Recognition memory on day 1 was low (40\%; Table 1B). After being instructed, on days 2 and 3 all BG EI participants had explicit information that the repeating sequence existed. Despite this subjective awareness, recognition memory was poor on day 2 (26\%; Table 1C). Prepractice sequence study on day 3 somewhat improved recognition (46\%), yet at the conclusion of day 3 it remained below chance (40\% at posttest). It is unclear why participants' recognition memory was occasionally below chance. It is possible that this test induced some distractor that caused participants to perform poorly on it. Unfortunately we are unable to address this possibility in the current study.

In addition, there was no relationship between improvements in tracking error, tracking time lag, or tracking accuracy and recognition memory for either group.

\section{DISCUSSION}

The present results demonstrate that providing explicit task information to BG and HC participants had opposite effects. Explicit information benefited implicit learning in the HC EI group, revealed by decreased tracking errors and a heightened temporal prediction of the target, but impaired these processes in participants with basal ganglia stroke. These effects were seen despite the fact that relatively few of those in the EI groups gained recognition memory for the repeating sequence, suggesting that the presence of explicit awareness may alter implicit learning. Previous work has demonstrated that manipulating explicit knowledge (directed attention) during practice changes performance (Shea and Wulf 1999). If indeed learning follows performance, then any operation that affects performance may alter learning as well. These data (Shea and Wulf 1999), in combination with ours, suggest that explicit information can influence both task performance and implicit learning.

Making the distinction between practice phase acquisition performance and implicit motor-sequence learning allowed us to reveal critical distinctions when considering the impact of explicit information on implicit learning. Classically defined, motor learning is "a set of processes associated with practice or experience leading to relatively permanent changes in the capability for movement" (Schmidt and Lee 1999). We distinguished between short-term performance related changes in behavior and long-term learning by administering a retention test on day 4. Hence, we determined that the detrimental effect of explicit information during acquisition was largely temporary and for the BG EI group diminishing at retention. Recall that significant differences were found between the BG EI and BG No-EI during the acquisition phase, but only a trend for these same differences was found during the day 4 retention phase. Conversely, the HC EI group demonstrated significantly greater changes in tracking error compared to the No-EI group that were only evident at retention, which suggests a long-term benefit of explicit information for the HC but not BG group. Employing a retention test

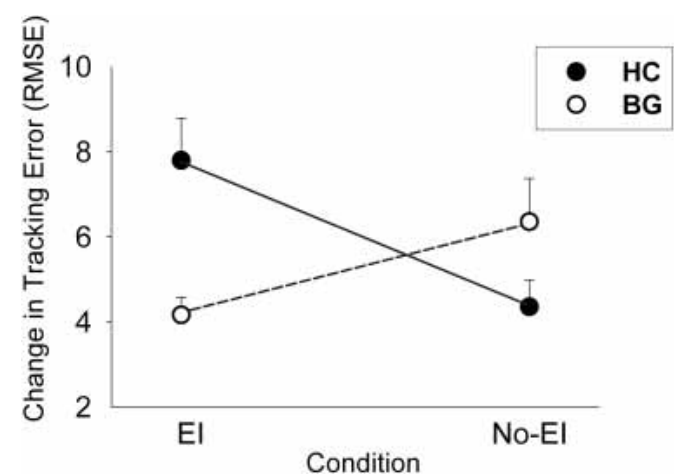

Figure 3 Learning at the retention test is demonstrated by larger change scores or greater change in tracking error for the learned sequence (relative to random). Explicit information had the opposite effect on the two groups. The HC El group was aided by explicit information, while the BG El group was not. HC groups are in solid circles and lines, BG groups are in open circles and dashed lines. Error bars are SEM. 

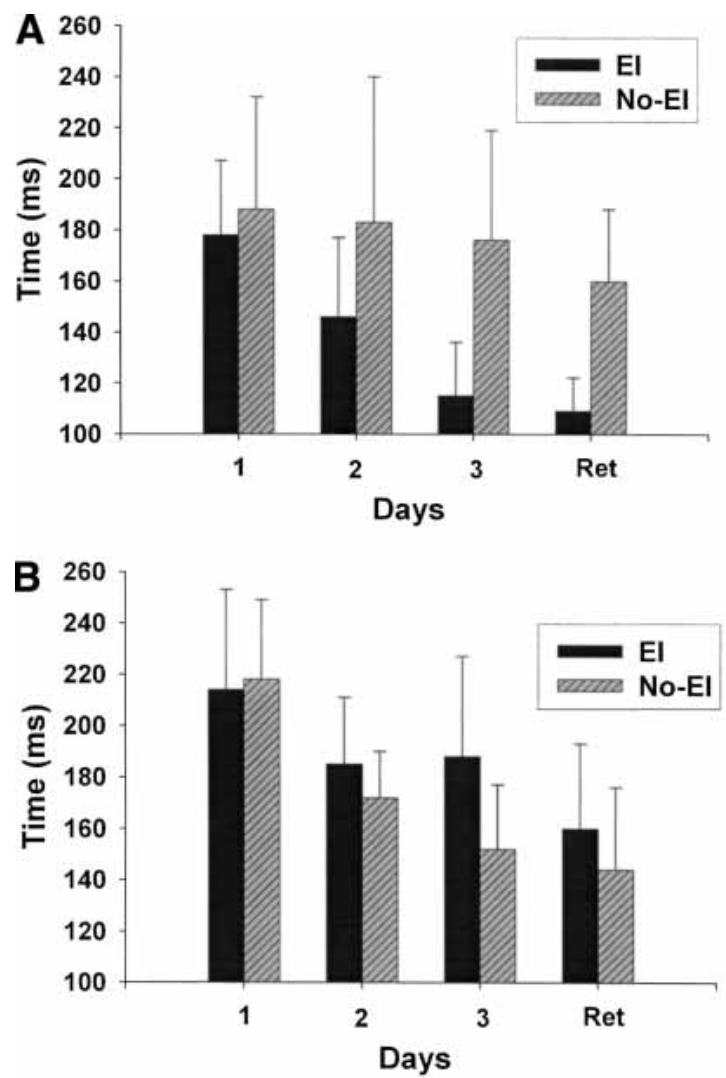

Figure 4 Change in time lag of tracking across three days of practice and at retention. El group is shown in solid bars; stripped bars represent No-El group data. Error bars are SEM. (A) Healthy Control Group. Both $\mathrm{HC}$ groups decreased their time lag across acquisition. However, the EI group decreased time lag of tracking significantly more than the No-El group. (B) Basal Ganglia Group. Across practice both BG El and No-El showed decreased time lag of tracking; however, this difference was not statistically reliable.

(administered after a one-day delay) allowed us to determine whether the effect of explicit information had a transient or more permanent effect on learning (Cahill et al. 2001).

Because our task allowed us to decompose movement into spatial and temporal components, we were able to assess with which factor(s) contributed to improved HC EI tracking performance. Both HC groups improved spatial tracking accuracy with practice. The largest benefit of explicit information was represented by the shortened tracking time lag for the HC EI group. Retention test tracking time lag for the HC EI group was so short (i.e., $109 \mathrm{~ms}+/-13.4$ ) that it could be considered anticipatory or predictive. The ability to predict the path of the target cursor demonstrates advance planning for upcoming movements in the sequence of repeated responses, and shows a beneficial aspect of explicit instruction for implicit CT learning.

The basal ganglia are normally critical for switching among different motor responses and thus in some fashion may represent the interface between explicit information and the implicit motor plan. Once a movement is started, individuals with PD have great difficulty stopping or transitioning to another response (Harrington and Haaland 1991), suggesting that damage to the basal ganglia disrupts the selection of responses. Additionally, several lines of evidence demonstrate that the basal ganglia are integrally involved in the advanced preparation of plans or programs for movement. For example, individuals with PD have difficulty using prior information about stimulus events to pre- pare movements (Stelmach et al. 1987; Jennings 1995; Harrington and Haaland 1998). This deficit is particularly striking when people with PD must construct a plan of action for a series of different movements (Harrington and Haaland 1991). In this instance, it appears that there is little benefit from giving extra time to prepare an action. Our finding that the BG EI participants were unable to use explicit information to plan for the tracking path of the CT sequence adds to this body of evidence, suggesting that the basal ganglia are critically involved in selecting motor responses, the advanced planning of movements, or both.

Our data are a part of an emerging theme in the motor skill learning literature suggesting that implicit and explicit learning can (1) be separated and operate in isolation or (2) impact and interact with one another (Willingham 2001). It cannot be assumed that the mixing of explicit and implicit is always helpful, and it appears that after basal ganglia stroke it is at least temporarily harmful. Our demonstration of a detrimental effect of explicit instructions is not new (Reber 1976; Green and Flowers 1991; Curran and Keele 1993; Boyd and Winstein 2003), but rarely has this work been extended into populations with neurologic damage. Long-ago Bliss (1892) and subsequently Boder (1935) described interference effects of explicit instructions on motor learning (Bliss 1892; Boder 1935). Disrupted learning after the adoption of conscious, experimenter suggested strategies has been demonstrated for both motor skills (Green and Flowers 1991; Verdolini-Marston and Balota 1994; Shea and Wulf 1999) and cognitive learning (Reber 1976; Poldrack et al. 2001). Pol-
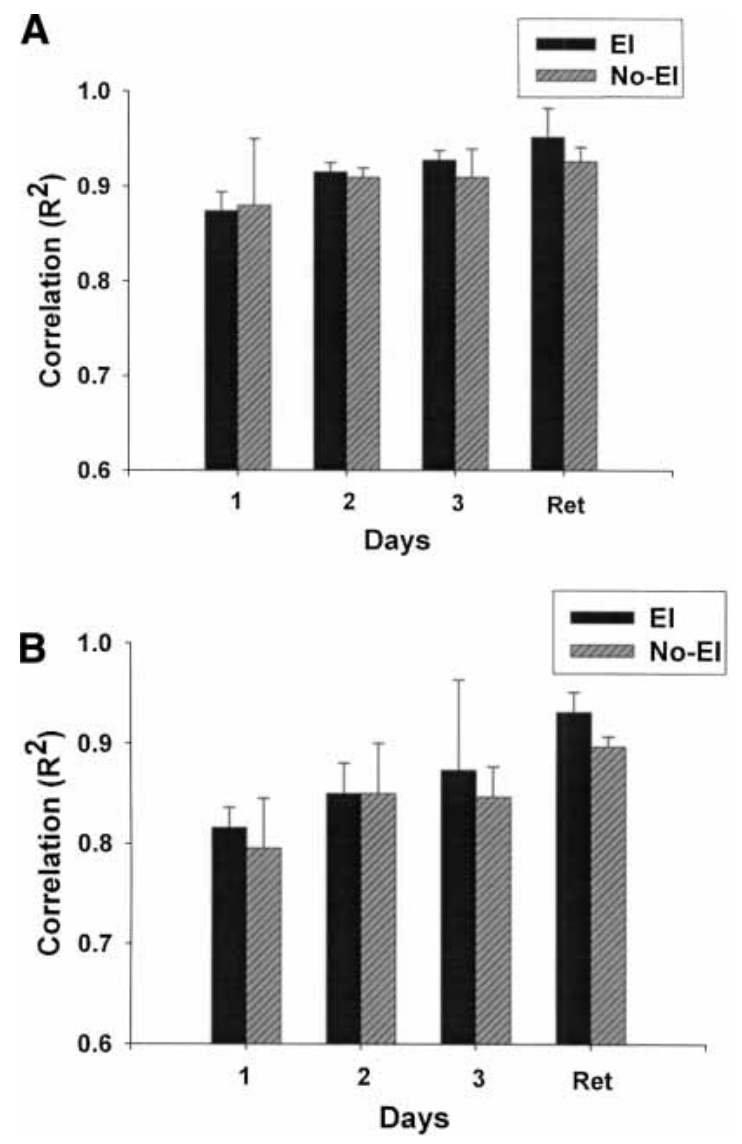

Figure 5 Change in tracking accuracy across three days of practice and at retention. El group is shown in solid bars; stripped bars represent No-EI group data. Error bars are SEM. All participants improved their tracking accuracy with practice and there were no group differences. $(A)$ Healthy Control Groups, (B) Basal Ganglia Groups. 
Table 1. Explicit Knowledge

\begin{tabular}{|c|c|c|c|}
\hline & $\begin{array}{l}\text { Subjective } \\
\% \text { noticed }\end{array}$ & $\begin{array}{c}\text { Sequence repeat } \\
\% \text { correct }\end{array}$ & $\begin{array}{l}\text { Recognition } \\
\% \text { correct }\end{array}$ \\
\hline \multicolumn{4}{|c|}{ A. No-El groups (postretention test) } \\
\hline $\mathrm{HC}$ & $0(0 / 5)$ & 20.0 & $\begin{array}{l}66.0^{* *} \\
(14.9)\end{array}$ \\
\hline BG & $0(0 / 5)$ & 0.0 & $\begin{array}{c}46.0 \\
(6.6)\end{array}$ \\
\hline \multicolumn{4}{|c|}{ B. El groups Day 1 (postpractice) } \\
\hline $\mathrm{HC}$ & $0(0 / 5)$ & 20.0 & $\begin{array}{c}33.3 \\
(8.0)\end{array}$ \\
\hline BG & $0(0 / 5)$ & 20.0 & $\begin{array}{c}40.0 \\
(16.9)\end{array}$ \\
\hline \multicolumn{4}{|c|}{ C. El groups Days 2 and 3} \\
\hline & \multicolumn{3}{|c|}{$\begin{array}{l}\text { Recognition } \\
\% \text { correct }\end{array}$} \\
\hline & $\begin{array}{l}\text { Postpractice } \\
\text { day } 2\end{array}$ & $\begin{array}{l}\text { Prepractice } \\
\text { day } 3\end{array}$ & $\begin{array}{l}\text { Postpractice } \\
\text { day } 3\end{array}$ \\
\hline $\mathrm{HC}$ & $\begin{array}{l}46.6 \\
(12.3)\end{array}$ & $\begin{array}{l}60.0 \\
(12.3)\end{array}$ & $\begin{array}{c}73.3 \\
(14.9)\end{array}$ \\
\hline BG & $\begin{array}{c}26.6 \\
(8.0)\end{array}$ & $\begin{array}{c}46.6 \\
(12.3)\end{array}$ & $\begin{array}{c}40.0 \\
(12.3)\end{array}$ \\
\hline
\end{tabular}

(A) Explicit knowledge for the No-El groups which was tested following the retention test on day 4. (B) Explicit knowledge for the $\mathrm{El}$ groups on day 1. (C) El group explicit testing was performed at the end of the second day of practice.

A pretest of explicit knowledge was administered the beginning of day three and repeated at the conclusion of practice for the El groups. All participants in the El groups were at least explicitly aware of the existence of a repeating sequence; recognition memory varied. Mean (standard error of the mean).

**Below $50 \%$ represents performance worse than change on recognition tests.

drack et al. (2001) showed that during classification learning the implicit and explicit systems may compete with one another. This is due to incompatible demands during learning; the need for access to flexible knowledge maintained by the medial temporal lobe stands at odds with the necessity of fast, automatic responses supported by the striatum. Arbitrating between these systems during learning normally induces rapid, reciprocal changes in the engagement of the medial temporal lobe compared with the striatum. Our data raise the possibility that damage to even a portion of the striatum disrupts these processes and affects implicit learning.

We asked, what happens then when the function of the implicit learning and memory system is disrupted by damage to the striatum? We expected that implicit learning after basal ganglia stroke would be aided by the provision of explicit information. This assumption proved to be incorrect. Our findings suggest that the BG EI participants attempted to apply an overt control strategy based on explicit information that resulted in the production of a less efficient movement pattern. Thus, for the BG group, explicit instructions appeared to interfere with the development of a correct and accurate strategy for success. These data and others (Wulf and Weigelt 1997) suggest that sometimes the cognitive demand of instructions may disrupt the formation of the implicit motor plan. This may occur because the rules necessary for successful task completion are not ones that can be expressed explicitly. Participants were being told to search for and use rules that they were not likely to find (Reber 1976) and not likely to find useful (Green and Flowers 1991). However, this only partially explains our data. The superior performance of the HC EI compared to the HC No-EI group suggests that another mechanism better explains the BG EI group's failure to benefit from explicit instruction.

During motor skill learning the basal ganglia may operate to coordinate multiple neural processes (Poldrack et al. 1999; Willingham et al. 2002). Numerous reciprocal connections exist between cortical regions and the basal ganglia, including the frontal cortex (Cavada and Goldman-Rakic 1991; Middleton and Strick 1997). There are at least five neuroanatomically separate, reciprocal basal ganglia-thalamocortical circuits, which allow the basal ganglia to have a widespread effect on cortical function (Alexander et al. 1986). The "motor" circuit, which is comprised of the putamen, thalamus, Supplementary Motor Area (SMA), and Premotor Cortex (PMC), is thought to most directly affect movement (Selemon and Goldman-Rakic 1985; Alexander et al. 1986). Yet, multiple "complex" circuits have been delineated, including one composed of interconnections between the caudate, thalamus, and dorsolateral prefrontal cortex (Middleton and Strick 1997). The complexity of the neuroanatomical interconnections between the cerebral cortex and basal ganglia suggest that their combined action can facilitate high-level integrative functions and modify action plans. Ideally situated to influence a wide range of learning tasks, activity in the fronto-striatal network has been associated with working memory load (Braver et al. 1997; Rypma et al. 1999). Converging evidence for this view stems from reports of disrupted working memory function in individuals with Parkinson's disease (Owen 1997). Our data advance this conceptualization; we suggest that providing explicit information may have induced an unmanageable working memory load and disrupted learning in participants with basal ganglia stroke.

We also considered whether our data could be explained by the possibility that stroke in the basal ganglia altered function in other areas of the brain. Although we cannot totally rule out this possibility, we believe that it is an unlikely explanation of our results. Indeed, the basal ganglia are highly interconnected with multiple brain regions (Middleton and Strick 1997) and damage to them undoubtedly has distant and widespread effects. However, our BG groups had similar lesion profiles (see Fig. 1) but demonstrated disparate ability during CT implicit learning, with the only difference being the provision of explicit information. One interesting detail of the present work is the use of the ipsilesional or less affected arm for implicit task practice. Deficient implicit learning after practice with the ipsilesional upper extremity suggests that normally bilateral basal ganglia somehow participate in this process. Indeed, strict contralateral activation of the basal ganglia during unimanual tasks has not been reported in neuroimaging work (Rao et al. 1997; Rypma et al. 1999). Taken together with our data, these results indicate that after unilateral basal ganglia damage, global functioning may be impaired.

\section{Conclusions}

Several conclusions may be drawn from our data. First, for healthy participants, explicit information appears to benefit implicit learning of the CT task. Second, damage to the basal ganglia disrupts the capacity for explicit information to constructively influence the formation of an implicit motor plan. In fact, it appears that after basal ganglia stroke explicit information is less helpful in the development of a motor plan than is discovering a motor solution using the implicit system alone. This may be due to the increased demand placed on working memory by explicit information. Others have reported basal ganglia activity in association with loading working memory; it appears that after damage the basal ganglia cannot accommodate this function. Thus, the integrity of the basal ganglia may be a crucial factor in de- 
termining the efficacy of explicit task information during implicit motor-sequence learning.

\section{MATERIALS AND METHODS}

\section{Participants}

Ten individuals who were at least 6 mo postunilateral stroke affecting the basal ganglia were recruited; 10 age-matched volunteers without any brain damage served as a healthy control group. All participants were right-hand dominant (determined by participant selfreport) and did not present with any evidence of dementia (26 or greater on the Mini-Mental State exam). Participants were excluded if they had any acute medical conditions, uncorrected vision loss, previous history of psychiatric admission, history of multiple strokes, transient ischemic attacks, or extensive cortical white matter disease. Individuals with stroke were recruited from the outpatient clinical services at the University of Southern California Healthcare Consultation Center, Rancho Los Amigos National Rehabilitation Center, and the South Bay Stroke support group. Individuals in the healthy control group were recruited from the local community. All participants signed an approved institutional informed consent form as well as a medical records release form prior to enrollment.

By random designation, participants were divided into either the EI or No-EI groups. There were no significant differences in age, education, MMSE or Fugl-Meyer scores between groups (Table 2).

\section{Lesion Location}

Prior to inclusion in this study an existing magnetic resonance image or computed tomography scan was obtained with written consent, and used to confirm a stroke affecting the basal ganglia. To illustrate the extent of damage each lesion was reconstructed using MRIcro software (Rorden 2003). Damaged regions were transformed onto a standard template which allowed the overlap of individual lesions into group data. Because side of lesion was not a variable of interest we normalized all lesions to the left and overlaid them (Fig. 1). The focus of overlap for both BG EI and BG No-EI groups was the putamen (Talairach coordinates BG EI -21, 1, 12; BG No-EI -21, 6, 11; Talairach and Tournoux 1988).

\section{Task and Procedure}

A lightweight lever was attached to a frictionless vertical axle, and secured to a table parallel to the floor. A linear potentiometer attached to the transducer at the base of the vertical axle recorded the analog signal that was converted to digital by a National Instruments A/D board (shielded multifunction I/O board, \#PCI-6024E) and sampled at $200 \mathrm{~Hz}$.

A target cursor (white $\mathrm{X}$ ) was visible on a black background as it moved from left to right across the screen (30 seconds total; $1.1 \mathrm{~cm} / \mathrm{sec}$; LabView software, National Instruments, Inc.). The task was to track the vertical path of the target with movements of the lever. Participants sat in front of the monitor with their arm resting on the lever and made arm motions from 0 to approximately $90^{\circ}$ of internal rotation with the start position at $45^{\circ}$; participant movements appeared as a green open square.

Unknown to the participants, the middle third of each tracking pattern was repeated and identical across practice and retention. This pattern was constructed using the polynomial equation as described by Wulf and Schmidt (1997) with the following general form:

$$
\begin{aligned}
f(x)= & b_{0}+a_{1} \sin (x)+b_{1} \cos (x)+a_{2} \sin (2 x)+b_{2} \cos (2 x) \\
& +\ldots+a_{6} \sin (6 x)+b_{6} \cos (6 x)
\end{aligned}
$$

The middle (repeated) segment was constructed by using the same coefficients for every trial ${ }^{4}$ (Wulf and Schmidt 1997). The first and third segments of the tracking pattern were generated randomly using coefficients ranging from 5.0 to -5.0 . A different random sequence was used for both the first and third segments for every trial. However, to ensure uniformity the same random tracking patterns were practiced by all of the participants. In each third of the tracking pattern there were 10 separate reversals in the direction of internal or external shoulder rotation.

The trajectories of the target and participants' movements did not leave a trail and thus, participants could not visualize the entire target pattern. Participants practiced 50 trials (5 blocks; 10 trials/block) each day under identical conditions. This procedure was repeated for $3 \mathrm{~d}$ (150 trials total) to ensure adequate acquisition practice (Boyd and Winstein 2001). To better separate performance effects from more permanent changes in behavior associated with learning, a retention test consisting of one block of continuous tracking was given on a separate fourth day. Instructions to track "as accurately as possible" were given daily. For all tracking trials the BG group used the arm ipsilateral to brain damage; the $\mathrm{HC}$ group was matched for arm use.

\section{Experimental Manipulation of Explicit Information}

\section{Explicit Information Groups}

Across the $3 \mathrm{~d}$ of practice, participants in the EI groups were progressively given explicit information of the task. On day 1, no explicit information was provided. Explicit knowledge was tested at the conclusion of practice day 1 for all EI participants. At the beginning of day 2, participants were explicitly instructed that there was a portion of the tracking task that repeated on every trial. From this point forward all participants in the EI groups' explicitly knew of the repeating sequence. Explicit knowledge (recognition memory) was tested again at the end of practice-day 2. At the beginning of day 3 , participants were explicitly informed of the location and composition of the sequence using a pictorial representation of the repeating sequence. Participants were asked to study, without physically practicing, the picture representation of the sequence. A pretest of explicit knowledge (recognition memory) was administered when participants indicated readiness (participants took 5-10 min to study). Following practice on day 3, explicit knowledge was reassessed. On retention test day 4, no explicit instructions or reminders were provided.

${ }^{4} b_{0}=2.0, a_{1}=-4.0, b_{1}=3.0, a_{2}=-4.9, b_{2}=-3.6, a_{3}=3.9, b_{3}=4.5$, $a_{4}=0.0, b_{4}=1.0, a_{5}=-3.8, b_{5}=-0.5, a_{6}=1.0$, and $b_{6}=2.5$ 


\section{No-Explicit Information Groups}

In contrast, participants in the No-EI groups were asked to track as accurately as possible and not given any indication of the existence of the repeating sequence. If they verbalized having noticed or asked about a sequence, the investigator remained neutral, providing no direct answer or response. Assessment of explicit knowledge occurred at the conclusion of day 4, after completion of the retention test.

\section{Explicit Testing}

Evaluation of explicit knowledge proceeded by subjectively asking participants if they had noticed anything about the task. Noticing a repeating segment triggered a request to locate it in the overall pattern (beginning, middle, or end). When participants failed to notice a repeating pattern they were informed of its existence and asked to guess where it was (Wulf and Schmidt 1997). Testing of subjective explicit awareness of the repeating sequence occurred on day 1 for the EI groups and on day 4 for the No-EI groups. Tests for recognition memory were administered by playing either the entire true sequence or one of two false tracking sequences on the computer screen and asking participants (yes/no) whether they recognized them.

\section{Outcome Measures}

Motor performance was evaluated across practice and retention in two ways. Our primary analysis considered changes in rootmean-squared error (RMSE) which reflects overall tracking errors in the kinematic pattern and is the average difference between the target pattern and participant movements. ${ }^{5}$ This score was calculated separately for random and repeating segments on every tracking trial and averaged by block (every 10 trials). Calculation of change in RMSE between the repeated and random sequences reflects sequence-specific learning. This measure was used to evaluate reductions in tracking errors across practice and at retention. Random tracking performance was characterized by the last block of random sequence performance on day 1 .

In our second analysis, we decomposed movement traces into spatial and temporal elements to determine the effect of EI and basal ganglia stroke on these two components of our tracking task. A time series analysis (TSA) was used to deconstruct tracking patterns into measures of spatial accuracy and the time lag between the kinematic patterns and the target. In the TSA, the tracking pattern from the repeated sequence of the trial was serially correlated with the target pattern (both 2000 data points) until a maximum correlation coefficient was achieved. Correlation coefficients $\left(\mathrm{R}^{2}\right)$ reflect the spatial accuracy of tracking performance. The distance (number of samples) that tracking data were moved (or slid) along the target data array to achieve the maximum correlation coefficient represents the time lag of tracking. Thus, time lag of tracking was converted to milliseconds for data analysis (samples to achieve maximum $\mathrm{r}$ multiplied by 5 $\mathrm{ms})$, and represents temporal distance from the target or temporal error.

\section{Statistical Analyses}

Acquisition performance was assessed using a three-factor analysis of variance (ANOVA; Group [HC, BG] by Information [EI, No-EI] by Day $[1,2,3])$ with repeated measures correction for Day. Subsequent, post hoc tests were performed to identify the locus of significant interactions. These separately examined information of the sequence (Information by Day ANOVA) and betweengroup differences (Group by Day ANOVA).

Retention test data reflect implicit motor-sequence learning. The magnitude of RMSE change at the retention test was evalu-

$$
5
$$

$$
\mathrm{RMSE}=\sum_{i=1}^{n}\left\{\left(x_{i}-T_{i}\right)^{2} / n\right\}^{1 / 2}
$$

$x_{i}=$ participant's position in degrees at time $1, T_{i}=$ target position at time 1, $n=$ the number of samples for the participant's trajectory array. ated with a two-factor ANOVA (Group by Information). Separate, post hoc one factor Information (EI, No-EI) and group (HC, BG) ANOVAs were performed to determine whether explicit information or participant population explained any significant interactions. These analyses were repeated for each dependent measure (RMSE, time lag, and tracking accuracy).

\section{ACKNOWLEDGMENTS}

The publication costs of this article were defrayed in part by payment of page charges. This article must therefore be hereby marked "advertisement" in accordance with 18 USC section 1734 solely to indicate this fact.

\section{REFERENCES}

Agid, Y. 1993. Mechanism of nerve-cell death in neurodegenerative disorders with a special reference to Parkinsons disease. Comptes Rendus des Seances de la Societe de Biologie et de Ses Filiales 187: 37-46. Alexander, G.E., DeLong, M.R., and Strick, P.L. 1986. Parallel organization of functionally segregated circuits linking basal ganglia and cortex. In Ann. Rev. Neurosci. (ed. W.M. Cowan), pp. 357-381. Society for Neuroscience, Washington, D.C.

Bliss, C.B. 1892. Investigations in reaction time and attention. Studies from the Yale Psychology Laboratory 1: 1-55.

Boder, D.P. 1935. The influence of concomitant activity and fatigue upon certain forms of reciprocal hand movements and its fundamental components. Comparative Psychology Monographs 11.

Boyd, L.A. and Winstein, C.J. 2001. Implicit motor-sequence learning in humans following unilateral stroke: The impact of practice and explicit knowledge. Neurosci. Lett. 298: 65-69.

. 2003. The impact of explicit information on implicit motor-sequence learning following middle cerebral artery area stroke. Phys. Ther. 83: 976-989.

Braver, T.S., Cohen, J.D., Nystrom, L.E., Jonides, J., Smith, E.E., and Noll, D.C. 1997. A parametric study of prefrontal cortex involvement in human working memory. Neuroimage 5: 49-62.

Cahill, L., McGaugh, J.L., and Weinberger, N.M. 2001. The neurobiology of learning and memory: Some reminders to remember. Trends Neurosci. 24: 578-581.

Cavada, C. and Goldman-Rakic, P.S. 1991. Topographic segregation of corticostriatal projections from posterior parietal subdivisions in the macaque monkey. Neuroscience 42: 683-696.

Curran, T. and Keele, S.W. 1993. Attentional and nonattentional forms of sequence learning. J. Experi. Psychol. Learn. Mem. Cognit. 19: $189-202$.

Doyon, J., Gaudreau, D., Laforce Jr., R., Castonguay, M., Bedard, P.J. Bedard, F., and Bouchard, J.P. 1997. Role of the striatum, cerebellum, and frontal lobes in the learning of a visuomotor sequence. Brain Cogn. 34: 218-245.

Doyon, J., Laforce Jr., R., Bouchard, G., Gaudreau, D., Roy, J., Poirier, M., Bedard, P.J., Bedard, F., and Bouchard, J.P. 1998. Role of the striatum, cerebellum and frontal lobes in the automatization of a repeated visuomotor sequence of movements. Neuropsychologia 36: 625-641.

Glisky, E.L. and Schacter, D. 1987. Acquisition of domain-specific knowledge in organic amnesia: Training for computer-related work. Neuropsychologia 25: 893-906.

Gomez-Beldarrain, M., Garcia-Monco, J.C., Rubio, B., and Pascual-Leone, A. 1998. Effect of focal cerebellar lesions on procedural learning in the serial reaction time task. Exp. Brain Res. 120: 25-30.

Green, T.D. and Flowers, J.H. 1991. Implicit versus explicit learning processes in a probabilistic, continuous fine-motor catching task. $J$. Mot. Behav. 23: 293-300.

Harrington, D.L. and Haaland, K.Y. 1991. Sequencing in Parkinson's disease: Abnormalities in programming and controlling movement. Brain 114: 99-115.

. 1998. Sequencing and timing operations of the basal ganglia. In Timing of behavior: Neural, psychological and computational perspectives, (eds. D.A. Rosenbaum and C.E. Collyer), pp. 35-61. MIT Press, Cambridge, MA.

Holthoff-Detto, V.A., Kessler, J., Herholz, K., Bonner, H., Peitrzyk, U. Wurker, M., Ghaemi, M., Wienhard, K., Wagner, R., and Heiss, W.D. 1997. Functional effects of striatal dysfunction in Parkinson disease. Arch. Neurol. 54: 145-150.

Jackson, G.M., Jackson, S.R., Harrison, J., Henderson, L., and Kennard C. 1995. Serial reaction time learning and Parkinson's disease: Evidence for a procedural learning deficit. Neuropsychologia 33: 577-593.

Jennings, P.J. 1995. Evidence of incomplete motor programming in Parkinson's disease. J. Mot. Behav. 27: 310-324. 
Kastner, A., Hirsch, E.C., Herrero, M.T., Javoyagid, F., and Agid, Y. 1993. Immunocytochemical quantification of tyrosine-hydroxylase at a cellular-level in the mesencephalon of control subjects and patients with Parkinsons and Alzheimers disease. J. Neurochem. 61: $1024-1034$.

Klingberg, T., O'Sullivan, B.T., and Roland, P.E. 1997. Bilateral activation of fronto-parietal networks by incrementing demand in a working memory task. Cereb. Cortex 7: 465-471.

Knowlton, B.J., Mangels, J.A., and Squire, L.R. 1996. A neostriatal habit learning system in humans. Science 273: 1399-1402.

Middleton, F.A. and Strick, P.L. 1997. New concepts about the organization of basal ganglia outputs. In Advances in neurology. Basal ganglia and new surgical treatment of Parkinson's disease, (eds. J.A. Obeso et al.), pp. 57-68. Lipincott-Raven, New York.

Nissan, M.J. and Bullemer, P. 1987. Attentional requirements of learning: evidence form performance measures. Cognit. Psychol. 19: $1-32$.

Owen, A.M. 1997. The functional organization of working memory processes within human lateral frontal cortex: The contribution of functional neuroimaging. Eur. J. Neurosci. 9: 1329-1339.

Pascual-Leone, A., Grafman, J., Clark, K., Stewart, M., Massaquoi, S., Lou, J.S., and Hallett, M. 1993. Procedural learning in Parkinson's disease and cerebellar degeneration. Ann. Neurol. 34: 594-602.

Pohl, P.S., Winstein, C.J., and Onla-or, S. 1997. Sensory-motor control in the ipsilesional upper extremity after stroke. NeuroRehabilitation 9: 57-69, Corringendum, 9: 245-249.

Pohl, P.S., McDowd, J.M., Filion, D.L., Richards, L.G., and Stiers, W. 2001. Implicit learning of a perceptual-motor skill after stroke. Phys. Ther. 81: 1780-1789.

Poldrack, R.A., Prabhakaran, V., Seger, C.A., and Gabrieli, J.D.E. 1999. Striatal activation during acquisition of a cognitive skill. Neuropsychology 13: 564-574.

Poldrack, R.A., Clark, J., Pare-Blagoev, E.J., Shohamy, D., Moyano, J.C., Myers, C., and Gluck, M.A. 2001. Interactive memory systems in the human brain. Nature 414: $546-550$.

Rao, S.M., Bobholz, J.A., Hammeke, T.A., Rosen, A.C., Woodley, S.J., Cunningham, J.M., Cox, R.W., Stein, E.A., and Binder, J.R. 1997. Functional MRI evidence for subcortical participation in conceptual reasoning skills. NeuroReport 8: 1987-1993.

Reber, A.S. 1976. Implicit learning of synthetic languages: The role of instructional set. J. Exper. Psychol. Hum. Learn. Mem. 2: 88-94.

Reber, P.J. and Squire, L.R. 1998. Encapsulation of implicit and explicit memory in sequence learning. J. Cogn. Neurosci. 10: 248-263.

Rorden, C. 2003. MRIcro. http://www.cla.sc.edu/psyc/faculty/rorden/mricro.html.

Rypma, B., Prabhakaran, V., Desmond, J.E., Glover, G.H., and Gabrieli,
J.D. 1999. Load-dependent roles of frontal brain regions in the maintenance of working memory. Neuroimage 9: 216-226.

Schmidt, R.A. and Lee, T.D. 1999. Motor control and learning: A behavioral emphasis, $2 \mathrm{~d}$ ed., p. 264. Human Kinetics, Champaign, IL.

Scoville, W.B. and Milner, B. 2000. Loss of recent memory after bilateral hippocampal lesions. 1957. J. Neuropsych. Clin. Neurosci. 12: $103-113$.

Selemon, L.D. and Goldman-Rakic, P.S. 1985. Longitudinal topography and interdigitation of corticostriatal projections in the rhesus monkey. J. Neurosci. 5: 776-794.

Shea, C. and Wulf, G. 1999. Enhancing motor learning through external-focus instructions and feedback. Hum. Move. Sci. 18: $553-571$.

Shea, C.H., Wulf, G., Whitacre, C.A., and Park, J.H. 2001. Surfing the implicit wave. Q. J. Exp. Psychol. A 54: 841-862.

Stelmach, G.E., Worringham, C.J., and Strand, E.A. 1987. The programming and execution of movement sequences in Parkinson's disease. Int. J. Neurosci. 36: 55-65.

Talairach, J. and Tournoux, P. 1988. Co-planar stereotaxic atlas of the human brain. Thieme, New York.

Vakil, E., Kahan, S., Huberman, M., and Osimani, A. 2000. Motor and non-motor sequence learning in patients with basal ganglia lesions: the case of serial reaction time (SRT). Neuropsychologia 38: 1-10.

Verdolini-Marston, K. and Balota, D.A. 1994. Role of elaborative and perceptual integrative processes in perceptual-motor performance. $J$. Exp. Psychol. Learn. Mem. Cogn. 20: 739-749.

Willingham, D.B. 2001. Becoming aware of motor skill. Trends Cogn. Sci. 5: $181-182$.

Willingham, D.B., Salidis, J., and Gabrieli, J.D.E. 2002. Direct comparison of neural systems mediating conscious and unconscious skill learning. J. Neurophys. 88: 1451-1460.

Winstein, C.J., Pohl, P.S., Cardinale, C., Green, A., Scholtz, L., and Waters, C.S. 1996. Learning a partial-weight-bearing skill: Effectiveness of two forms of feedback. Phys. Ther. 76: 985-993.

Winstein, C.J., Merians, A.S., and Sullivan, K.J. 1999. Motor learning after unilateral brain damage. Neuropsychologia 37: 975-987.

Wulf, G. and Schmidt, R.A. 1997. Variability of practice and implicit motor learning. J. Exp. Psychol. Learn. Mem. Cogn. 23: 987-1006.

Wulf, G. and Weigelt, C. 1997. Instructions about physical principles in learning a complex motor skill: to tell or not to tell... Res. Q. Exerc. Sport 68: 362-367.

Received April 12, 2004; accepted in revised form June 9, 2004.

\section{Learning \& Memory}




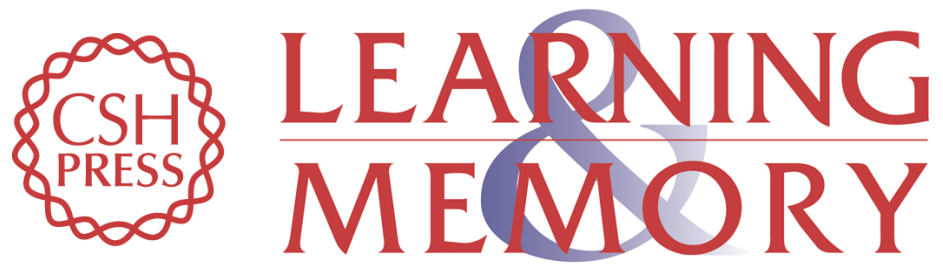

\section{Providing Explicit Information Disrupts Implicit Motor Learning After Basal Ganglia Stroke}

Lara A. Boyd and Carolee J. Winstein

Learn. Mem. 2004, 11:

Access the most recent version at doi:10.1101//m.80104

References This article cites 39 articles, 5 of which can be accessed free at:

http://learnmem.cshlp.org/content/11/4/388.full.html\#ref-list-1

License

Email Alerting Receive free email alerts when new articles cite this article - sign up in the box at the Service top right corner of the article or click here. 